\title{
Anaesthetic Considerations for Rationalizing Drug Use in the Operating Theatre: Strategies in a Singapore Hospital During COVID-19
}

\author{
Phui Sze Angie Au Yong ${ }^{1}$ (D) Charlene Wen Xian Kwa ${ }^{1} \cdot$ Xin Hui Diana Chan ${ }^{1}$
}

Accepted: 1 June 2020 / Published online: 12 June 2020

(C) Springer Nature Switzerland AG 2020

\begin{abstract}
COVID-19 patients in the critical care unit tend to have prolonged hospital stay requiring high doses of sedation and paralysis to treat acute respiratory distress syndrome, resulting in a shortage of these drugs. In our hospital, we have instituted strategies to rationalise drug and oxygen usage. This includes prioritising time-sensitive elective cases, reducing overall elective case load, favouring opioid-reduction strategies and usage of alternative anaesthetic agents not commonly used in ICU. Both intensive care physicians and anaesthesiologists have to cooperate on drug conservation as similar drugs are used in elective operating lists as in the ICU. Patient safety is of utmost importance and we should keep in mind some pitfalls and ethical concerns of these alternative strategies.
\end{abstract}

Keywords SARS-CoV-2 $\cdot$ COVID-19 $\cdot$ Intensive Care $\cdot$ Anesthesia $\cdot$ Drug $\cdot$ Rational Use $\cdot$ Conservation $\cdot$ Pandemic

\section{Introduction}

Coronavirus disease (COVID-19) was declared a pandemic by the World Health Organization [1] on 11 March 2020 and has infected more than 2 million people worldwide. Hospitals are facing an unprecedented surge in intensive care unit (ICU) patients because 5-10\% of COVID-19 patients deteriorate rapidly and require prolonged intensive care for acute respiratory distress syndrome [2]. These patients tend to require high doses of sedatives and paralytics. This has resulted in increasing worldwide demand for critical care drugs, including and not limited to sedatives, paralytics, opioids and vasoactive agents, especially to support ventilatory strategies for ARDS such as proning and paralysis [3].

During peace time in the pre COVID-19 era, hospital infection control adopts a general policy of discarding any portion of unused drug rather than risk contamination. Drug sharing across patients is prohibited. However, during a pandemic,

This article is part of the Topical Collection on Covid-19

Phui Sze Angie Au Yong

angie.au.yong.p.s@singhealth.com.sg

1 Division of Anesthesiology and Perioperative Medicine, Singapore General Hospital, 1 Hospital Drive, Singapore 169608, Singapore resource conservation becomes essential. There is a scarcity of literature advocating for this and a concern as to whether this comprises patient safety and recovery profile. The Faculty of Intensive Care Medicine and Specialist Pharmacy Service in the UK has introduced guides for ICUs on adaptations to minimise wastage for critical care medications during pandemic emergency pressures $[4,5]$. We describe our local strategies for anaesthesiologists in the operating theatre (OT) to rationalise drug use because the same drugs are commonly used in both ICU and OT, highlighting the pitfalls that deviating from routine practice may have on patient safety and recovery. These strategies were not a strict enforcement, but rather a guide in addition to usual anaesthetic considerations.

\section{Prioritising Surgeries and Drug Conservation}

At hospital level, elective cases were reduced to only essential time-sensitive cases such as cancer surgery as a healthy patient may end up consuming resources which may make a difference in outcome to the next critically-ill patient.

At the departmental level, pre-emptive planning of drugs needed for the elective lists was encouraged, as entire vial of drugs such as dexmedetomidine, propofol and midazolam are often discarded after using a small portion. There is a caution for cross-contamination and microbial activity especially for 
propofol. Dilution of drugs for patients transferring from OT to ICU were standardized so that re-dilution of drugs can be avoided, hence minimizing medication wastage.

\section{Regional Anaesthetic (RA) Techniques and Opioid-Sparing Strategies}

Rostered specialists from the department RA subspeciality interest group were available to provide technical support for RA during COVID. A guide for practical considerations was published to help standardise practice and reduce periprocedure COVID transmission risks [6]. Regional techniques can provide adequate anaesthesia in majority of limb and lower abdominal surgery, reduce sedative and opioid use and oxygen utilisation compared with general anaesthesia [7].

\section{Use of Alternative Agents and Considerations}

The pharmacy and ICU provided updates when drugs were low on supply to help in OT list planning. If general anaesthesia cannot be avoided, anaesthesiologists were encouraged to use other drugs not commonly used in ICU, e.g. ketamine, nitrous oxide, volatiles, thiopentone and etomidate. Oral premedications like lorazepam or gabapentin were used to reduce anaesthetic requirements. Multimodal analgesia was optimised with non-opioid adjuncts, such as intravenous (IV) lignocaine infusion, magnesium, dexamethasone, clonidine, ketamine and non-steroidal anti-inflammatory drugs (NSAIDS).

Total intravenous anaesthesia (TIVA) commonly requires large amounts of propofol and remifentanil infusions and was recommended to be avoided as much as possible for routine anaesthesia during COVID. If TIVA is deemed necessary, such as spine cases requiring neuromonitoring, or neurosurgery with raised intracranial pressure, modifications to the technique to reduce propofol usage were considered. This included using midazolam, dexmedetomidine or volatiles less than 1 MAC where appropriate. Once the need for neuromonitoring is over, maintenance of anaesthesia can be switched to volatiles rather than TIVA. Depth of anaesthesia monitoring was encouraged to avoid excessive anaesthetic use and hypotension requiring vasopressors.

\section{Oxygen Use during Anaesthesia}

Oxygen was recommended to be rationalised for patients with decent cardiorespiratory reserves. Other than during preoxygenation and extubation, minimal-to-low fresh gas flows were used. Inspired oxygen concentrations were titrated to less than $30 \%$ as long as the patient did not desaturate.
Down-titrating volatiles or using nitrous oxide near the end of surgery can aid in faster anaesthetic washout prior to extubation without the use of excessively high flows. Postoperative oxygen supplementation was titrated if needed rather than a blanket measure.

\section{Limitations and Potential Pitfalls}

While considering the use of alternative drugs for sedation and analgesia, anaesthesiologists should be aware of the potential pitfalls in the pharmacokinetics and pharmacodynamics of alternative agents. Use of less familiar or less titratable agents may result in delayed or less favourable emergence profiles, or unanticipated side effects. For example, thiopentone has less suppression of laryngeal reflexes than propofol and may cause increased coughing or bronchospasm on emergence, further increasing aerosolisation of respiratory particles. Etomidate has been shown to cause adrenal suppression even with just one dose [8], which may affect recovery outcomes if the patient is a presymptomatic carrier of Sars-CoV-2 [9], and turns COVID-positive later. Ketamine may raise BIS readings and confound the interpretation of depth of anaesthesia.

\section{Ethical Considerations}

In the process of modifying anaesthetic technique to conserve drugs, it is important to consider the risks of alternatives. Inadequate regional block necessitating conversion to general anaesthesia halfway through surgery may pose more harm from manipulating airway under less-than-ideal conditions. Opioid-sparing analgesia should not result in inadequate analgesia. Some anaesthesiologists may be uncomfortable in providing what they perceive to be substandard care by using alternatives. Hence, drug rationalisation is a more appropriate term than drug conservation, as every patient should receive tailored anaesthetic care based on their individual risk-benefit ratio. In patients with moderate to high risk, delivery of a safe anaesthetic with drugs that have favourable profiles should definitely supercede concerns of drug shortage.

\section{Conclusion}

To conclude, multidisciplinary coordination is needed to make a concerted effort for drug conservation during COVID-19 pandemic and allocating them to dire areas of need. Anaesthesiologists can contribute by meticulously planning ahead and modifying usual techniques without increased risks to the patient. 
Authors' Contributions PSA Au Yong drafted, contributed and reviewed final manuscript; CWX Kwa and XHD Chan contributed and reviewed final manuscript.

\section{Compliance with Ethical Standards}

Conflict of Interest The authors declare that they have no conflict of interest.

Ethical Approval Not required.

Informed Consent Not required.

\section{References}

1. World Health Organization. Statement on the second meeting of the International Health Regulations. Emergency committee regarding the outbreak of novel coronavirus (2019-nCoV). 2005. Available from URL: https://www.who.int/news-room/detail/30-01-2020statement-on-the-second-meeting-of-the-international-healthregulations-(2005)-emergency-committee-regarding-the-outbreakof-novel-coronavirus-(2019-ncov) (Accessed 13 April 2020).

2. Poston JT, Patel BK, Davis AM. Management of critically ill adults with COVID-19. JAMA. 2020. https://doi.org/10.1001/jama.2020. 4914.

3. Mezher M, Taylor NP. FDA reports shortage of sedation drug used for putting COVID-19 patients on ventilators [internet]. Available from URL: https://www.raps.org/news-and-articles/news-articles/ 2020/4/fda-reports-shortage-of-sedation-drug-used-for-put (Accessed 15 April 2020).
4. Faculty of Intensive Care Medicine, UK. Guidance on adaptations to standard UK critical care medication prescribing and administration practices during pandemic emergency pressures. Available from URL: https://icmanesthesiacovid-19.org/drug-demand-supplyadaptations-to-uk-critical-care-medication-prescribing (Accessed 13 April 2020).

5. National Health Service, Specialist Pharmacy Service, UK. COVID19 Guidance: Minimising Wastage of Critical Injectable Medicines. Available from URL: https://www.sps.nhs.uk/wp-content/uploads/ 2020/04/COVID-19-Guidance-Minimising-Wastage-of-CriticalInjectable-Medicines-Aseptics-v2.pdf (Accessed 13 April 2020).

6. Lie SA, Wong SW, Wong LT, Wong TGL, Chong SY. Practical considerations for performing regional anesthesia: lessons learned from the COVID-19 pandemic [published online ahead of print, 2020 Mar 24]. Considérations pratiques pour laréalisation de l'anesthésie régionale: les leçons tirées de la pandémie de COVID19 [published online ahead of print, 2020 Mar 24]. Can J Anaesth. 2020;67:1-8. https://doi.org/10.1007/s12630-020-01637-0.

7. Au Yong PSA, Chan DXH. Opioid reduction strategies are important for laparotomies during the covid-19 outbreak. Reg Anesth Pain Med. 2020:rapm-2020-101533. https://doi.org/10.1136/rapm-2020101533.

8. Chan CM, Mitchell AL, Shorr AF. Etomidate is associated with mortality and adrenal insufficiency in sepsis. Crit Care Med. 2012;40(11):2945-53. https://doi.org/10.1097/CCM. 0b013e31825fec26.

9. Wei WE, Li Z, Chiew CJ, Yong SE, Toh MP, Lee VJ. Presymptomatic transmission of SARS-CoV2 - Singapore, January 23-March 16, 2020. MMWR Morb Mortal Wkly Rep. 2020;69:411-5. https://doi.org/10.15585/mmwr.mm6914e1 ePub: 1.

Publisher's Note Springer Nature remains neutral with regard to jurisdictional claims in published maps and institutional affiliations. 